\title{
Intestinal endometriosis treated by laparoscopic surgery: case series of 5 patients
}

\author{
Hiroka Kondo ${ }^{*}$, Yasumitsu Hirano, Toshimasa Ishii, Kiyoka Hara, Nao Obara, Liming Wang, Masahiro Asari, \\ Takuya Kato and Shigeki Yamaguchi
}

\begin{abstract}
Background: Intestinal endometriosis is rare and most frequently involves the rectum and sigmoid colon.

Case presentation: Here, we report a case series of 5 patients who underwent laparoscopic resection for intestinal endometriosis. None of the patients developed postoperative complications, and all were discharged at 5-8 days after surgery. The diagnosis of intestinal endometriosis is difficult to obtain before surgery. Only 2 of 5 patients were diagnosed preoperatively. Among 1 of the 2 patients, the symptoms at the time of menstruation were obvious. In patients with submucosal tumors, the preoperative diagnosis can be difficult. Additional imaging examinations at the time of menstruation might be useful for obtaining a diagnosis. D2 dissections were performed for 3 patients, because malignancy could not be ruled out as a preoperative diagnosis. The surgical findings of 1 patient did not appear to be endometriosis. Surgery for intestinal endometriosis usually encounters advanced pelvic adhesions and fibrosis. For patients undergoing sigmoidectomy, the mean operative time was $152 \mathrm{~min}$ and mean blood loss was $10 \mathrm{~mL}$. For patients undergoing rectal resection, the mean operative time was $282 \mathrm{~min}$ and mean blood loss was $17 \mathrm{~mL}$. Two cases had severe pelvic adhesions, and the residual rectum could not be straightened. Therefore, sideto-side anastomosis was performed. For intestinal endometriosis surgery, flexible planning for the anastomosis method used for residual intestine should be undertaken.

Conclusion: Laparoscopic surgery for intestinal endometriosis was safe but technically difficult, because of fibrosis and adhesions. An accurate diagnosis should be attempted based on the clinical symptoms, imaging findings, and intraoperative findings. The method used for anastomosis should be decided on a case-by-case basis.
\end{abstract}

Keywords: Intestinal endometriosis, Laparoscopic surgery

\section{Background}

Intestinal endometriosis is rare. It is seen in about 10 to $30 \%$ of patients with endometriosis [1] and most frequently involves the rectum and sigmoid colon [2]. Here, we report a case series of 5 patients who underwent laparoscopic resection for intestinal endometriosis.

\footnotetext{
* Correspondence: hirokak@saitama-med.ac.jp

Department of Gastroenterological Surgery, Saitama Medical University International Medical Center, 1397-1 Yamane, Hidaka-shi, Saitama 350-1298, Japan
}

\section{Case report}

After a positive fecal occult blood test (FOBT), colonoscopy revealed an extramural mass in the sigmoid colon (Fig. 1), and the patient was referred to our hospital. Computed tomography (CT) revealed thickening of the wall of the sigmoid colon (Fig. 2). Our preoperative diagnosis was a malignant tumor such as gastrointestinal stromal tumor (GIST). We performed laparoscopic sigmoidectomy. The pouch of Douglas contained severe fibrosis. Side-to-side anastomosis was performed because straightening the rectum was difficult. 


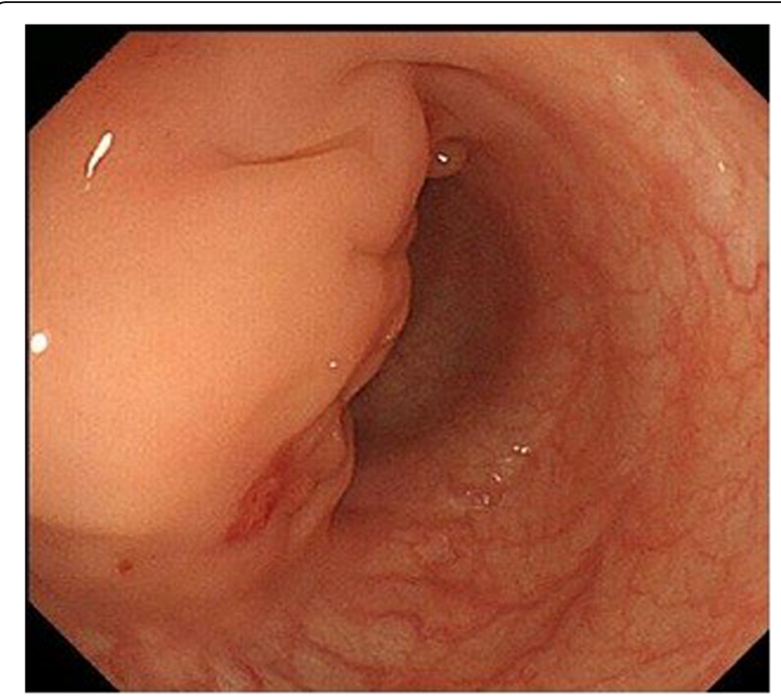

Fig. 1 Colonoscopy revealed an extramural mass associated with the sigmoid colon

\section{Case 2: a 48-year-old woman}

After a positive FOBT, and with a history of constipation, the patient underwent rectal examination, which revealed a palpable mass. A barium enema examination revealed a $40-\mathrm{mm}$ extramural mass in the rectum (Fig. 3), and the patient was referred to our hospital. A colonoscopy was negative for mucosal findings. Computed tomography (CT) and magnetic resonance imaging (MRI) revealed a 40-mm mass on the anterior wall of the rectum (Figs. 4 and 5, respectively). Our preoperative diagnosis was a malignant tumor such as GIST. We performed a low anterior laparoscopic resection and found severe fibrosis in the pouch of Douglas.

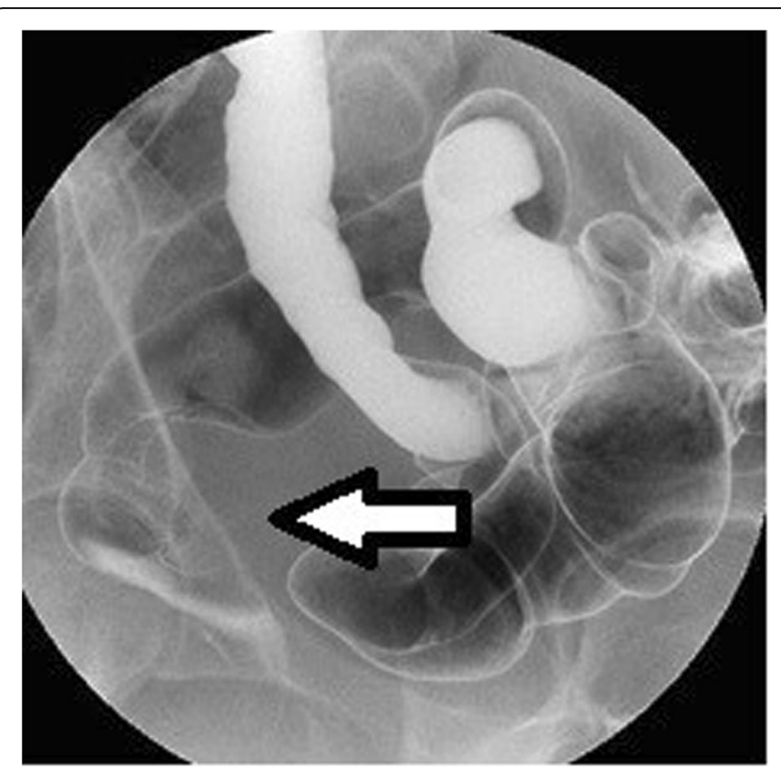

Fig. 3 A barium enema examination shows a 40-mm extramural mass in the rectum

\section{Case 3: a 50-year-old woman}

The patient presented at another hospital with a history of 2 to 3 months of abdominal pain. Abdominal ultrasonography revealed a mass at the end of the ileum, and she was referred to our hospital. A colonoscopy revealed a submucosal tumor at the end of the ileum, and we marked the location by a tattoo. CT was unremarkable, but MRI revealed a mass at the end of the ileum (Fig. 6). Our preoperative diagnosis was intestinal endometriosis because symptoms always appear at menstruation. A 30$\mathrm{mm}$ mass was found at the end of the ileum, and a partial small bowel resection and end-to-end anastomosis were performed.

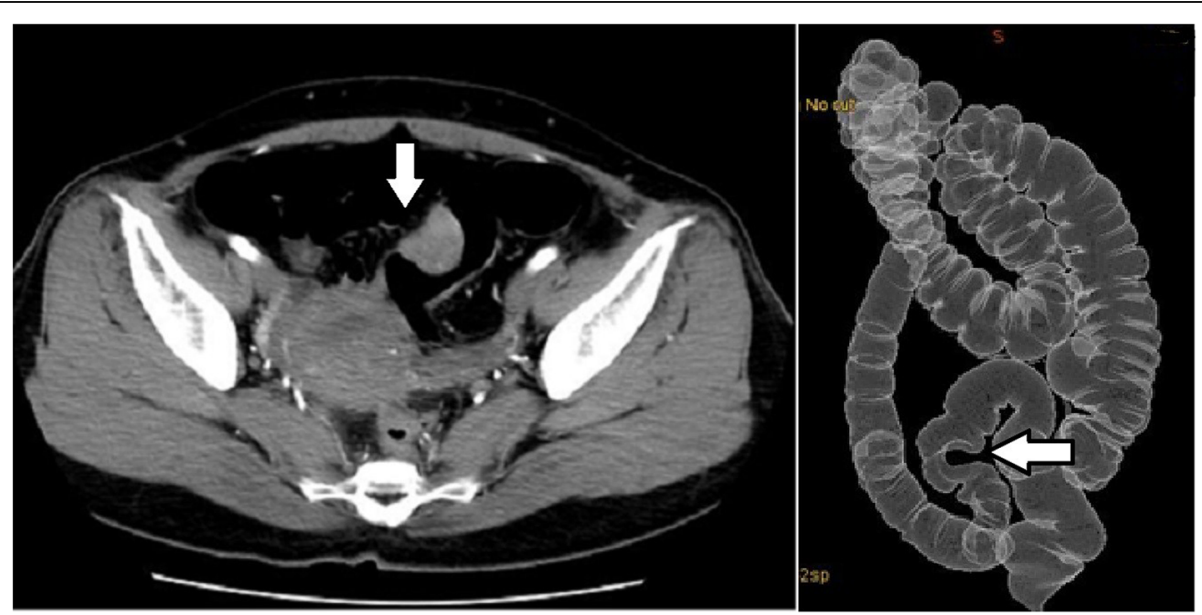

Fig. 2 Computed tomography (CT) reveals the thickened wall of the sigmoid colon (arrow), and CT colonography shows the deformed wall in the sigmoid colon (arrow) 


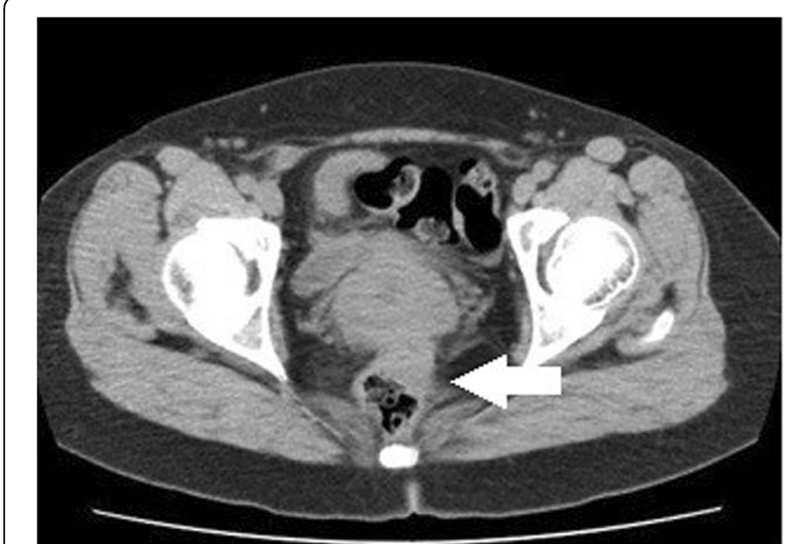

Fig. 4 Computed tomography shows the $40-\mathrm{mm}$ mass on the anterior wall of the rectum

\section{Case 4: a 37-year-old woman}

After a positive FOBT, colonoscopy revealed a $25-\mathrm{mm}$ submucosal tumor in the sigmoid colon (Fig. 7), and the patient was referred to our hospital. CT reveal a 20-mm mass in the sigmoid colon (Fig. 8). Our preoperative diagnosis was a malignant tumor such as GIST. We performed a high anterior laparoscopic resection and found severe fibrosis in the pouch of Douglas. We suspected endometriosis.

\section{Case 5: a 43-year-old woman}

The patient presented at another hospital with a 5-year history of constipation. A barium enema examination

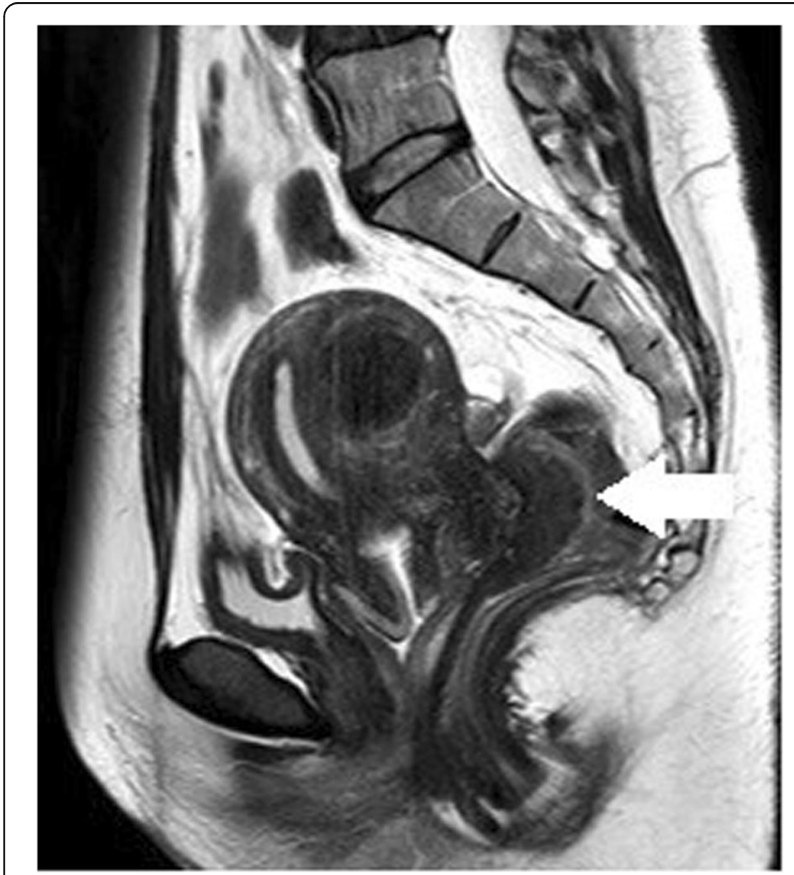

Fig. 5 Magnetic resonance imaging (MRI) shows a 40-mm mass on the anterior wall of the rectum

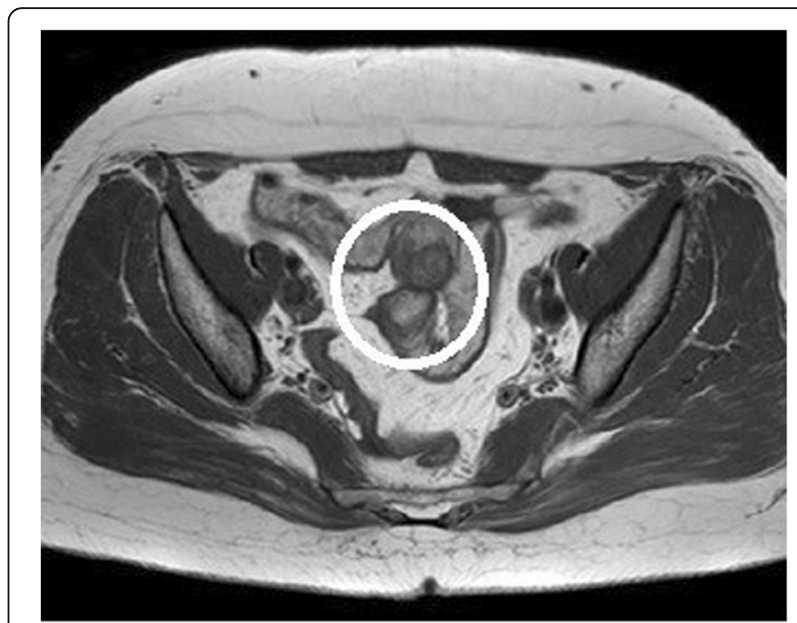

Fig. $6 \mathrm{MRI}$ shows a mass at the end of the ileum (circle)

revealed an extramural mass in the sigmoid colon (Fig. 9), and she was referred to our hospital. A colonoscopy revealed an extramural mass in the sigmoid colon, and the scope could not be passed due to stenosis. CT revealed the mass in the sigmoid colon (Fig. 10). The referral gynecologist had diagnosed endometriosis, and our preoperative diagnosis was intestinal endometriosis. We performed a laparoscopic sigmoidectomy and found severe fibrosis in the pouch of Douglas. Because straightening the rectum was difficult, a side-to-side anastomosis was performed.

Preoperative biopsies were performed in two of the five patients, but none were diagnosed because they were biopsies from the mucosal surface. Although endoscopic ultrasound-guided fine needle aspiration (EUS-FNA) was

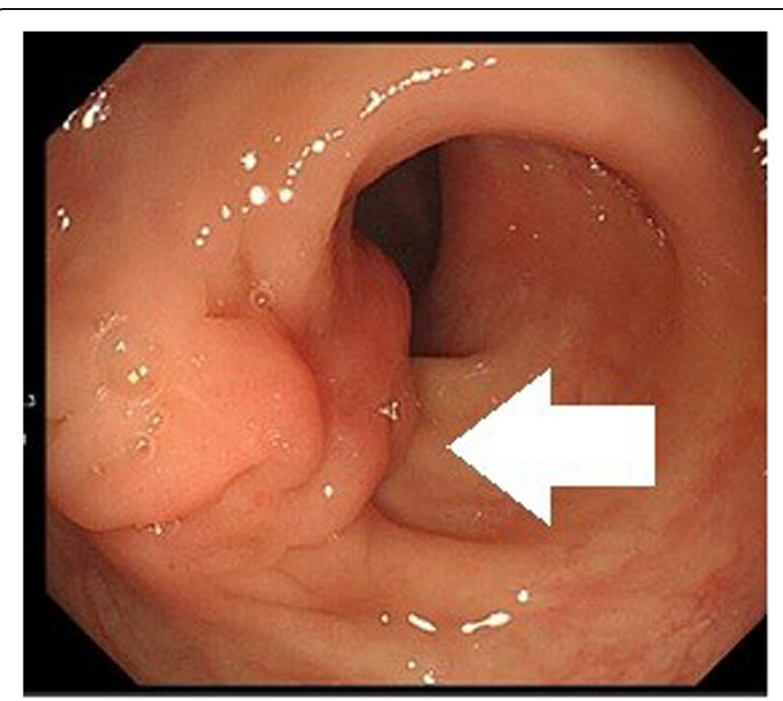

Fig. 7 Colonoscopy shows a 25-mm submucosal tumor in the sigmoid colon (arrow) 


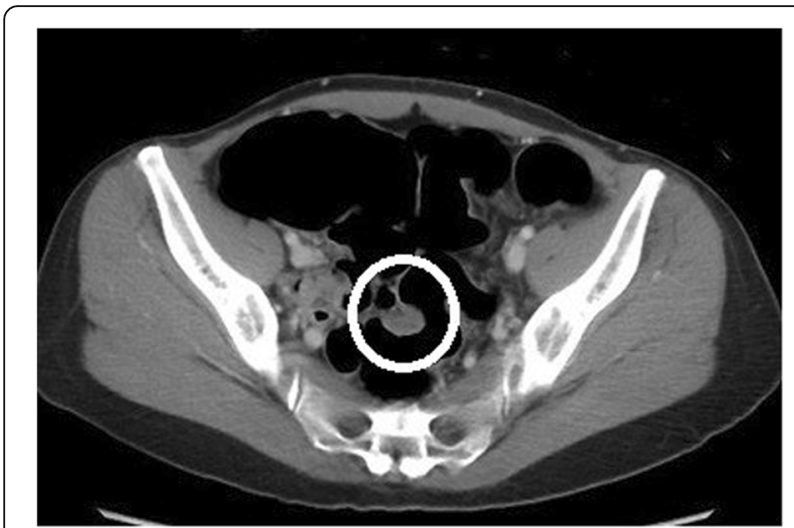

Fig. 8 Computed tomography shows a 20-mm mass in the sigmoid colon (circle)

considered in all cases, all cases were symptomatic and early surgery was requested, so EUS-FNA was not performed.

None of the patients developed postoperative complications, and all patients were discharged at 5-8 days after surgery.

\section{Discussion}

Endometriosis is a general term for endometrial tissue found outside the uterus and is present in 5 to $15 \%$ of women of reproductive age [3]. It has been reported that 12 to $37 \%$ of patients with endometriosis have intestinal endometriosis [1]. Ectopic endometrial tissue invades and grows in the intestinal tract, causing bleeding, stenosis, and adhesions. The most common site of this disease is said to be the sigmoid colon or rectum [2].

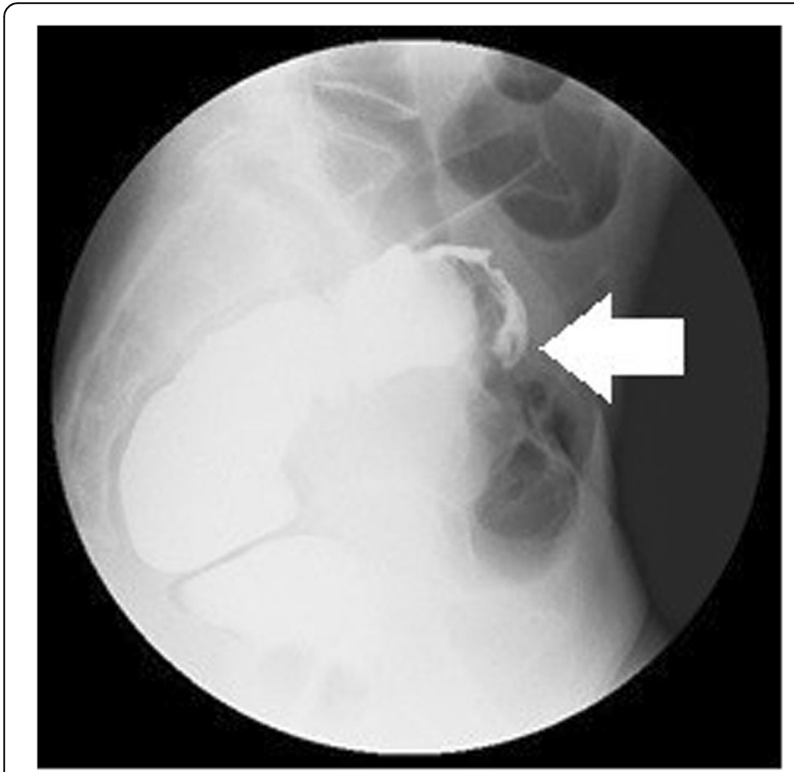

Fig. 9 A barium enema examination shows an extramural mass in the sigmoid colon (arrow)
Before surgery, intestinal endometriosis was diagnosed in 2 of the 5 patients. Otherwise, it was difficult to diagnose preoperatively. Among the 2 patients, the clarifying symptom of pain was present at the time of menstruation in only 1 patient.

In case 3, non-menstrual CT showed no findings at the terminal ileum, but MRI performed during menstruation showed a mass. Because of the different modalities, it is not possible to conclude based on this report alone, but it was also important to examine the difference between menstrual and non-menstrual images in diagnostic imaging of intestinal endometriosis. Although no reports on menstrual and nonmenstrual imaging have been found in previous reports, the accumulation of future cases is considered important. Obtaining a preoperative diagnosis appears to be difficult for patients with a submucosal lesion; however, Oliveira et al. reported that intestinal endometriosis should be considered in the differential diagnosis of patients with submucosal tumors of the colon [4]. If intestinal endometriosis is established as part of the differential diagnosis, a preoperative diagnosis should not be difficult.

D2 dissection was performed for case nos. 1, 2, and 4; because malignancy could not be ruled out preoperatively. Tumors arising from intestinal endometriosis are rare and are collectively referred to as endometriosisassociated intestinal tumor (EAIT) [5]. Malignant transformation can develop in some cases [6], and metastatic lymph nodes have been observed [7]. Previous reports indicate that $78.7 \%$ of endometrial malignancies occur in the ovaries and $21.3 \%$ outside the ovaries. Of the latter, the pelvis accounted for $5.7 \%$, the rectovaginal septum $4.3 \%$, the colorectum $4.3 \%$, and the small intestine $0.5 \%$ [8].

There have been reports that endometrial tissue has also been found in regional lymph nodes at the time of the resection of the intestine involved with endometriosis; however, the relationship between lymph nodes containing endometrial lesions and the malignant transformation of endometrial lesions in the intestine has not been clarified, and carcinomas associated with intestinal endometriosis are rare [9, 10]. Some believe that lymphadenectomy is not so effective for endometriosis [11]. If there is no suspicion of a malignant tumor, lymph node dissection is unnecessary, even if the lymph nodes are involved. If malignancy cannot be ruled out, excision of the intestinal tract with lymph node dissection should be considered.

The surgical findings of 1 of our 5 cases did not suggest endometriosis. The surgical findings of surgery for intestinal endometriosis often include advanced pelvic adhesions and fibrosis. These findings 


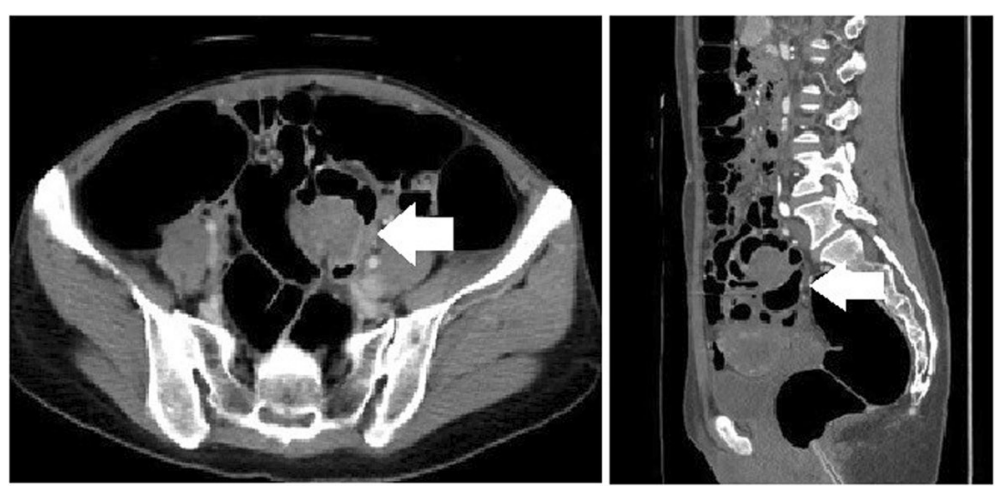

Fig. 10 Computed tomography shows the mass in the sigmoid colon (arrow)

are thought to be caused by endometrial tissue, which undergoes fibrosis, and metaplasia of smooth muscle extending throughout the muscular layer to the subserosal layer of the intestinal tract and repeatedly causing bleeding and inflammation along with the menstrual cycle [12].

Rocha et al. have evaluated laparoscopic surgery in endometriosis surgery with colectomy and report that it is also effective in alleviating abdominal symptoms and preserving fertility [13]. For patients undergoing sigmoidectomy, the mean operative time was $152 \mathrm{~min}$ and mean blood loss was $10 \mathrm{~mL}$. For patients undergoing rectal resection, the mean operative time was $282 \mathrm{~min}$ and mean blood loss was 17 $\mathrm{mL}$. Two cases had severe pelvic adhesions, and the residual rectum could not be straightened. Therefore, side-to-side anastomosis was performed. For intestinal endometriosis surgery, flexible planning for the anastomosis method for a residual intestine should be undertaken.

\section{Conclusion}

Laparoscopic surgery for intestinal endometriosis was safe but technically difficult, because of fibrosis and adhesions. An accurate diagnosis should be attempted based on the clinical symptoms, imaging findings, and intraoperative findings. The method used for anastomosis should be decided on a caseby-case basis.

\section{Abbreviations}

CT: Computed tomography; EUS-FNA: Endoscopic ultrasound-guided fineneedle aspiration; FOBT: Fecal occult blood test; GIST: Gastrointestinal stromal tumor; MRI: Magnetic resonance imaging

\section{Acknowledgements}

None.

\section{Authors' contributions}

HK and YH wrote the manuscript. SY supervised the case. All other authors reviewed the manuscript. All authors read and approved the final manuscript.
Funding

No funding was received for this case series

Availability of data and materials

Data sharing is not applicable to this article, since datasets were neither generated nor analyzed for the case series.

Ethics approval and consent to participate Not applicable.

\section{Consent for publication}

Written informed consent was obtained from the patients for the publication of this report.

\section{Competing interests}

The authors declare that they have no competing interests.

Received: 20 January 2020 Accepted: 26 February 2020

Published online: 10 March 2020

\section{References}

1. Orbuch $\mathbb{I K}$, Reich $\mathrm{H}$, Orbuch $\mathrm{M}$, et al. Laparoscopic treatment of recurrent small bowel obstruction secondary to ileal endometriosis. J Minim Inacasive Gynecol. 2007;14:113-5.

2. Mcafee $\mathrm{CH}$, Greer $\mathrm{HL}$. Intestinal endometriosis: a report of 29 cases and a survey of the literature. J Obstet Gynecol Br Comm. 1960:67:539-55.

3. Lu PY, Ory SJ. Endometriosis: current management. Mayo Clin Proc. 1995; 170:453-63.

4. Oliveira A, Costa M, Amaro P, et al. Appendiceal submucosal mass as presentation of intestinal endometriosis. Asian J Endosc Surg. 2015;8(3):337-

5. Slavin RE, Krum R, Van Dinh T, et al. Endometriosis-associated intestinal tumors: a clinical and pathological study of 6 cases with a review of the literature. Hum Pathol. 2000;31:456-63.

6. Yang H, Gu JJ, Qi Y, et al. Endometrioid adenocarcinoma of the rectovaginal septum with invasion of the rectum: a case report and review of literature. World J Surg Oncol. 2019;17(1):206

7. Tsuchiya T, Hiramatsu K, Otsuji H, et al. A resected case of endometriosis of the rectum with pararectal lymph node metastatic involvement. J Japan Surg Assoc. 2011;72(2):418-22.

8. Hoang CD, Boettcher AK, Jessurun J, et al. An unusual rectosigmoid mass: endometrioid adenocarcinoma arising in colonic endometirosis: case report and literature review. Am Surg. 2005;71(8):694-7.

9. Darai E, Dubernard G, Coutant C, et al. Randomized trial of laparoscopically assisted versus open colorectal resection for endometriosis: morbidity, symptoms, quality of life, and fertility. Ann Surg. 2010;251:1018-23.

10. Practice Committee of the American Society for Reproductive Medicine; Society of Reproductive Surgeons. Pathogenesis, consequences, and control of peritoneal adhesions in gynecologic surgery. Fertil Steril. 2007;88:21-6. 
11. Kinuko N, Takashi U, Tatsuya M, et al. Five cases of laparoscopic bowel resection for intestinal endometriosis. Jpn J Gastroenterol Surg. 2016;49(8): 762-71.

12. Itoga $T$, Takeuchi $H$, Matsumoto $T$, et al. Fibrosis and smooth muscle metaplasia in rectovesinal endometriosis. Pathol Int. 2003;53:371-5.

13. Rocha AM, Albuquerque MM, Schmidt EM, et al. Late impact of the laparoscopic treatment of deep infiltrating endometriosis with segmental colorectal resection. Arq Bras Cir Dig. 2018;31(4):e1406.

\section{Publisher's Note}

Springer Nature remains neutral with regard to jurisdictional claims in published maps and institutional affiliations.

Submit your manuscript to a SpringerOpen ${ }^{\mathcal{O}}$ journal and benefit from:

- Convenient online submission

- Rigorous peer review

- Open access: articles freely available online

- High visibility within the field

- Retaining the copyright to your article

Submit your next manuscript at $\boldsymbol{\wedge}$ springeropen.com 